\title{
Regular vector lattices of continuous functions and Korovkin-type theorems-Part II
}

\author{
by \\ Francesco Altomare and Mirella Cappelletti Montano (Bari)
}

\begin{abstract}
By applying the results of the first part of the paper, we establish some Korovkin-type theorems for continuous positive linear operators in the setting of regular vector lattices of continuous functions. Moreover, we present simple methods to construct Korovkin subspaces for finitely defined operators and for the identity operator and we determine those classes of operators which admit finite-dimensional Korovkin subspaces. Finally, we give a Korovkin-type theorem for continuous positive projections.
\end{abstract}

1. Introduction. In this second part of the paper, by using the results of [3], we establish some Korovkin-type theorems for continuous positive linear operators on particular lattices of continuous functions on a locally compact Hausdorff space $X$, called regular vector lattices on $X$, which we have introduced in [3, Definition 2.2].

In the sequel, we keep the same notations used in [3].

Let $(E, \tau)$ be a regular vector lattice on a locally compact Hausdorff space $X$. In $[3,(3.11)$ and (3.12)] we have introduced suitable envelopes for functions of $E$ and we have studied their properties. Here, given a continuous positive linear operator $T$ from $E$ into $E$, we shall use these properties in order to characterize those subspaces $H$ of $E$ which are Korovkin subspaces in $E$ for $T$ and $\tau$, i.e. for every $\tau$-equicontinuous net $\left(L_{i}\right)_{i \in I}^{\leq}$of positive linear operators from $E$ into $E$ satisfying $\lim _{i \in I} \leq L_{i}(h)=T(h)$ for every $h \in H$ in $(E, \tau)$, one has $\lim _{i \in I} \leq L_{i}(f)=T(f)$ for every $f \in E$ in $(E, \tau)$.

Our results generalize those of many previous papers. For example, H. Bauer and K. Donner (see [8]) and F. Altomare and M. Campiti (see [1]) have considered the case where $E=C_{0}(X, \mathbb{R})$, and W. Roth has studied in [17] the case where $E$ is a weighted function space (see [15], [16]) and $T$ is the identity operator on $E$.

2000 Mathematics Subject Classification: 47B38, 47B65, 41A35, 46E05.

Key words and phrases: positive operator, positive projection, finitely defined operator, Korovkin-type approximation theorem, vector lattice of continuous functions. 
Moreover, M. W. Grossman (see [13]) (resp., H. Bauer in [6], [7] for the identity operator) has studied similar problems in the context of adapted spaces of continuous functions in the sense of Choquet (see [9], [14], [18]). Finally in [12] (see also [10], [11]) H. Flösser, R. Irmisch and W. Roth have determined, with a partially different approach, Korovkin-type theorems in the setting of sequentially complete $M$-spaces with topological orthogonal systems.

We also present simple methods to construct Korovkin subspaces for particular positive linear operators, called finitely defined operators, and for the identity operator. Moreover, we exhibit examples of finite-dimensional Korovkin subspaces for these operators and we show that finitely defined operators are the only ones which admit finite-dimensional Korovkin subspaces.

We also prove that the Korovkin subspaces for the identity operator are Korovkin subspaces for every $(\tau, \tau)$-continuous lattice homomorphism on $E$.

Finally, we present a Korovkin-type theorem for a continuous positive projection $T$. Denoting by $H_{T}$ the range of such a projection, we characterize the Choquet boundary of $H_{T}$ in terms of the given projection and we determine particular Korovkin subspaces for this operator. This last part of the paper is a generalization of a previous work of the authors (see [2]) in the framework of adapted spaces (see also [1, Sect. 3.3]).

2. Korovkin closures for positive linear operators. Let $(E, \tau)$ be a regular vector lattice on a locally compact Hausdorff space $X$ (see [3, Definition 2.2]) and denote by $\mathcal{U}_{\tau}$ the neighborhood base of $(E, \tau)$ consisting of absolutely convex sublattices defined in [3, Definition 2.2, property (i)]. Moreover, consider $T \in \mathcal{L}_{\tau}^{+}(E)$ (see [3, (3.9)]). In this section, we characterize those subspaces of $E$ (if any) such that every $\tau$-equicontinuous net of positive linear operators from $E$ into $E$ converging to $T$ on them, automatically converges to $T$ on the whole space $E$.

To this end, we recall that a net $\left(L_{i}\right)_{i \in I}^{\leq}$of positive linear operators from $E$ into $E$ is said to be equicontinuous if for every neighborhood $V$ of the origin of $E$ there exists a neighborhood $U$ of the origin of $E$ such that $L_{i}(f) \in V$ for every $f \in U$ and $i \in I$.

In the next definition we introduce the subspace we are mainly interested in (see [1], [10]-[12], [17]).

Definition 2.1. Let $(E, \tau)$ be a regular vector lattice on a locally compact Hausdorff space $X, H$ a subspace of $E$, and $T \in \mathcal{L}_{\tau}^{+}(E)$. The subspace of $E$ defined by 
$\operatorname{Kor}(H)_{\tau, T}:=\left\{f \in E \mid\right.$ if $\left(L_{i}\right)_{i \in I}^{\leq}$is a $\tau$-equicontinuous net of positive linear operators from $E$ into $E$ satisfying

$$
\begin{aligned}
& \lim _{i \in I} \leq L_{i}(h)=T(h) \text { in }(E, \tau) \text { for every } h \in H, \text { then } \\
& \left.\lim _{i \in I} \leq L_{i}(f)=T(f) \text { in }(E, \tau)\right\}
\end{aligned}
$$

is said to be the Korovkin closure of $H$ in $E$ for the operator $T$ and the topology $\tau$.

Moreover, $H$ is said to be a Korovkin subspace in $E$ for the operator $T$ and the topology $\tau$ if $\operatorname{Kor}(H)_{\tau, T}=E$.

We now give a characterization of $\operatorname{Kor}(H)_{\tau, T}$.

TheOREM 2.2. Let $(E, \tau)$ be a regular vector lattice on a locally compact Hausdorff space $X, H$ a subspace of $E$, and $T \in \mathcal{L}_{\tau}^{+}(E)$. Then

$\operatorname{Kor}(H)_{\tau, T}=\widehat{H}_{\tau, T}$

$$
=\left\{f \in E \mid \int f d \mu=T(f)(x) \text { for every } x \in X \text { and } \mu \in M_{\tau, x}^{T}(H)\right\},
$$

where $\widehat{H}_{\tau, T}$ and $M_{\tau, x}^{T}(H)$ are defined as in (3.14) and (3.15) of [3], respectively.

Proof. The second equality is obvious, by Corollary 3.6 of [3].

We proceed to prove that, if $f \in \operatorname{Kor}(H)_{\tau, T}$, then $\int f d \mu=T(f)(x)$ for every $x \in X$ and $\mu \in M_{\tau, x}^{T}(H)$. To this end, given $x \in X$ and $\mu \in M_{\tau, x}^{T}(H)$, choose a compact neighborhood $Q_{0}$ of $x$, and denote by $\mathcal{B}$ the fundamental system of neighborhoods of $x$ consisting of all (relatively compact) neighborhoods of $x$ contained in $Q_{0}$.

For every $Q \in \mathcal{B}$ there exists a function $q_{Q} \in C_{\mathrm{c}}(X, \mathbb{R})$ such that $0 \leq$ $q_{Q} \leq 1, q_{Q}(x)=1$ and $q_{Q}(y)=0$ for every $y \notin Q$; then, for every $Q \in \mathcal{B}$, set

$$
\varphi_{Q}:=\inf \left\{q_{Q}, q_{Q_{0}}\right\}
$$

and consider the positive linear operator $L_{Q}$ defined by

$$
L_{Q}(g):=\left(\int g d \mu\right) \varphi_{Q}+T(g)-T(g) \varphi_{Q} \quad(g \in E) .
$$

Since $C_{\mathrm{c}}(X, \mathbb{R}) \subset E$ (see [3, Definition 2.2, property (iii)]), each $L_{Q}$ acts from $E$ to $E$. Moreover, $\left(L_{Q}\right)_{Q \in \mathcal{B}}^{\supset}$ is a $\tau$-equicontinuous net. Indeed, for every $Q \in \mathcal{B}$ we get

$$
\left|L_{Q}(g)\right| \leq\left|\int g d \mu\right| \varphi_{Q_{0}}+|T(g)| .
$$

Choose $V \in \mathcal{U}_{\tau}$ and $W \in \mathcal{U}_{\tau}$ such that $W+W \subset V$; since $T$ is $(\tau, \tau)$ continuous, there exists $U \in \mathcal{U}_{\tau}$ such that $T(g) \in W$ for every $g \in U$ and thus $|T(g)| \in W$. Moreover, there exists $\lambda>0$ such that $\lambda \varphi_{Q_{0}} \in W$. Accordingly, there exists $U^{\prime} \in \mathcal{B}$ such that $\left|\int g d \mu\right| \leq \lambda$ for every $g \in U^{\prime}$. 
Therefore, for every $g \in U \cap U^{\prime}$ and $Q \in \mathcal{B}$,

$$
\left|L_{Q}(g)\right| \in W+W \subset V .
$$

This shows that $\left(L_{Q}\right)_{Q \in \mathcal{B}}^{\supset}$ is equicontinuous.

We proceed to show that $\lim _{V \in \mathcal{B}} \supset L_{V}(h)=T(h)$ for every $h \in H$ in $(E, \tau)$. First, note that if $Q \in \mathcal{B}$, then for every $h \in H$,

$$
\left|L_{Q}(h)-T(h)\right| \leq|T(h)-T(h)(x)| \varphi_{Q} .
$$

Moreover, if $V \in \mathcal{U}_{\tau}$, then by [3, Proposition 2.8], there exists a neighborhood $P \subset Q_{0}$ of $x$ such that

$$
|T(h)-T(h)(x)| \varphi \in V
$$

for every $\varphi \in C_{\mathrm{c}}(X, \mathbb{R})$ with $0 \leq \varphi \leq 1$ and $\operatorname{supp}(\varphi) \subset P$. Choose a compact neighborhood $Q_{1}$ of $x$ such that $Q_{1} \subset P$. Then $Q_{1} \in \mathcal{B}$ and for every $Q \in \mathcal{B}$ with $Q \subset Q_{1}$, we get $\operatorname{supp}\left(\varphi_{Q}\right) \subset Q_{1} \subset P$; thus,

$$
\left|L_{Q}(h)-T(h)\right| \leq|T(h)-T(h)(x)| \varphi_{Q} \in V .
$$

Accordingly, $\lim _{V \in \mathcal{B}} \supset L_{V}(h)=T(h)$ in $(E, \tau)$. Therefore, $\lim _{V \in \mathcal{B}} \supset L_{V}(f)=T(f)$ in $(E, \tau)$, so that, since $L_{V}(f)(x)=\int f d \mu$ for every $V \in \mathcal{B}$, it follows that $\int f d \mu=T(f)(x)$.

We now proceed to show that if $f \in \widehat{H}_{\tau, T}$, then $f \in \operatorname{Kor}(H)_{\tau, T}$. To this end, given a $\tau$-equicontinuous net $\left(L_{i}\right)_{i \in I}^{\leq}$of positive linear operators from $E$ into $E$ satisfying $\lim _{i \in I} \leq L_{i}(h)=T(h)$ for every $h \in H$ in $(E, \tau)$, fix $V \in \mathcal{U}_{\tau}$ and consider $W \in \mathcal{U}_{\tau}$ such that $W+W+W+W+W+W \subset V$. Then, by assumption, there exists $U \in \mathcal{U}_{\tau}$ with $U \subset W$ such that

$$
L_{i}(g) \in W, \quad T(g) \in W
$$

for every $g \in U$ and $i \in I$. According to Theorem 3.7 of [3], there exist $k_{1}, \ldots, k_{n}, k_{1}^{\prime}, \ldots, k_{m}^{\prime} \in H$ such that

$$
\left(f-k_{p}\right)^{+} \in U, \quad\left(k_{q}^{\prime}-f\right)^{+} \in U
$$

for every $p=1, \ldots, n$ and $q=1, \ldots, m$ and

$$
\inf _{1 \leq p \leq n} T\left(k_{p}\right)-\sup _{1 \leq q \leq m} T\left(k_{q}^{\prime}\right) \in U .
$$

Set $u:=\sup _{1 \leq p \leq n}\left(f-k_{p}\right)^{+} \in U$ and $v:=\sup _{1 \leq q \leq m}\left(k_{q}^{\prime}-f\right)^{+} \in U$; from (3) it follows that $L_{i}(u), L_{i}(v), T(u), T(v) \in W$ for every $i \in I$. Furthermore, since for every $p=1, \ldots, n$ and $q=1, \ldots, m$,

$$
k_{q}^{\prime}-v \leq k_{q}^{\prime}-\left(k_{q}^{\prime}-f\right)^{+} \leq f \leq k_{p}+\left(f-k_{p}\right)^{+} \leq k_{p}+u,
$$

it follows that for every $i \in I$,

$$
L_{i}\left(k_{q}^{\prime}\right)-L_{i}(v) \leq L_{i}(f) \leq L_{i}\left(k_{p}\right)+L_{i}(u)
$$


and

$$
T\left(k_{q}^{\prime}\right)-T(v) \leq T(f) \leq T\left(k_{p}\right)+T(u) ;
$$

hence

$$
\sup _{1 \leq q \leq m} L_{i}\left(k_{q}^{\prime}\right)-L_{i}(v) \leq L_{i}(f) \leq \inf _{1 \leq p \leq n} L_{i}\left(k_{p}\right)+L_{i}(u)
$$

and

$$
\sup _{1 \leq q \leq m} T\left(k_{q}^{\prime}\right)-T(v) \leq T(f) \leq \inf _{1 \leq p \leq n} T\left(k_{p}\right)+T(u) .
$$

Consequently,

$$
\begin{aligned}
& L_{i}(f)-T(f) \leq \inf _{1 \leq p \leq n} L_{i}\left(k_{p}\right)+L_{i}(u)-\sup _{1 \leq q \leq m} T\left(k_{q}^{\prime}\right)+T(v) \\
& =\inf _{1 \leq p \leq n} L_{i}\left(k_{p}\right)-\inf _{1 \leq p \leq n} T\left(k_{p}\right)+\inf _{1 \leq p \leq n} T\left(k_{p}\right)-\sup _{1 \leq q \leq m} T\left(k_{q}^{\prime}\right)+L_{i}(u)+T(v) \\
& \leq \sum_{p=1}^{n}\left|L_{i}\left(k_{p}\right)-T\left(k_{p}\right)\right|+\inf _{1 \leq p \leq n} T\left(k_{p}\right)-\sup _{1 \leq q \leq m} T\left(k_{q}^{\prime}\right)+L_{i}(u)+T(v) .
\end{aligned}
$$

By a similar reasoning, we also get

$$
\begin{aligned}
& T(f)-L_{i}(f) \leq \inf _{1 \leq p \leq n} T\left(k_{p}\right)+T(u)-\sup _{1 \leq q \leq m} L_{i}\left(k_{q}^{\prime}\right)+L_{i}(v) \\
& =\inf _{1 \leq p \leq n} T\left(k_{p}\right)-\sup _{1 \leq q \leq m} T\left(k_{q}^{\prime}\right)+\sup _{1 \leq q \leq m} T\left(k_{q}^{\prime}\right)-\sup _{1 \leq q \leq m} L_{i}\left(k_{q}^{\prime}\right)+T(u)+L_{i}(v) \\
& \leq \inf _{1 \leq p \leq n} T\left(k_{p}\right)-\sup _{1 \leq q \leq m} T\left(k_{q}^{\prime}\right)+\sum_{q=1}^{m}\left|T\left(k_{q}^{\prime}\right)-L_{i}\left(k_{q}^{\prime}\right)\right|+T(u)+L_{i}(v) .
\end{aligned}
$$

Accordingly,

$$
\begin{aligned}
\left|L_{i}(f)-T(f)\right| & \leq \sum_{p=1}^{n}\left|L_{i}\left(k_{p}\right)-T\left(k_{p}\right)\right|+\sum_{q=1}^{m}\left|L_{i}\left(k_{q}^{\prime}\right)-T\left(k_{q}^{\prime}\right)\right| \\
& +\left|\inf _{1 \leq p \leq n} T\left(k_{p}\right)-\sup _{1 \leq q \leq m} T\left(k_{q}^{\prime}\right)\right|+L_{i}(u)+T(v)+T(u)+L_{i}(v) .
\end{aligned}
$$

Fix now $U^{*} \in \mathcal{U}_{\tau}$ such that

$$
\underbrace{U^{*}+\cdots+U^{*}}_{n+m \text { times }} \subset U
$$

Since $\lim _{i \in I} \leq L_{i}(h)=T(h)$ in $(E, \tau)$ for every $h \in H$, there exists $i_{0} \in I$ such that for every $i \in I$ with $i \geq i_{0}$,

$$
L_{i}\left(k_{p}\right)-T\left(k_{p}\right) \in U^{*} \quad \text { and } \quad L_{i}\left(k_{q}^{\prime}\right)-T\left(k_{q}^{\prime}\right) \in U^{*}
$$

for every $p=1, \ldots, n$ and $q=1, \ldots, m$; hence

$$
\sum_{p=1}^{n}\left|L_{i}\left(k_{p}\right)-T\left(k_{p}\right)\right|+\sum_{q=1}^{m}\left|L_{i}\left(k_{q}^{\prime}\right)-T\left(k_{q}^{\prime}\right)\right| \in U \subset W .
$$


Consequently, for every $i \in I$ with $i \geq i_{0}$, we get

$$
L_{i}(f)-T(f) \in W+W+W+W+W+W \subset V,
$$

and this completes the proof.

In the following result, we characterize those subspaces $H$ of $E$ which are Korovkin subspaces in $E$ for $T$ and $\tau$. Its proof, taking Corollary 3.6 of [3] into account, is straightforward.

COROLlary 2.3. Under the assumptions of Theorem 2.2, $H$ is a Korovkin subspace in $E$ for $T$ and $\tau$ if and only if $\partial_{\tau, H}^{T} X=X$, where $\partial_{\tau, H}^{T} X$ is defined by $[3,(3.17)]$.

3. Korovkin subspaces for finitely defined operators. In this section we describe several methods to construct Korovkin subspaces for a class of positive linear operators, namely finitely defined operators. This class of operators was studied in [1, Section 3.4] in the framework of continuous functions which vanish at infinity; by using similar methods, we extend those results to arbitrary regular vector lattices.

Moreover, we exhibit some examples of finite-dimensional Korovkin subspaces for finitely defined operators and we prove that, in some sense, they are the only class of positive linear operators which admit finite-dimensional Korovkin subspaces.

First, we introduce the following definition.

DEFinition 3.1. Let $(E, \tau)$ be a regular vector lattice on a locally compact Hausdorff space $X$. Every positive linear operator $T: E \rightarrow E$ of the form

$$
T(f):=\sum_{i=1}^{n} \lambda_{i}\left(f \circ \varphi_{i}\right) \quad(f \in E),
$$

where $\lambda_{i} \in C(X, \mathbb{R}), \lambda_{i} \geq 0, \varphi_{i}: X \rightarrow X$ is continuous and $\lambda_{i}\left(f \circ \varphi_{i}\right) \in E$ for every $f \in E(1 \leq i \leq n)$, is said to be a finitely defined operator of order $n$.

In general, a finitely defined operator $T$ with a representation (3.1) is not necessarily $(\tau, \tau)$-continuous, unless $\tau$ is the topology $\tau_{\mathrm{c}}$ of uniform convergence on compact subsets of $X$ or the topology $\tau_{\mathrm{s}}$ of pointwise convergence on $X$.

For a fixed finitely defined operator $T \in \mathcal{L}_{\tau}^{+}(E)$ with a representation (3.1), we denote by $n(x)$ the number of distinct points of the set $\left\{\varphi_{1}(x), \ldots, \varphi_{n}(x)\right\}(x \in X)$; without loss of generality, we assume that, up to a suitable permutation, these points are $\left\{\varphi_{1}(x), \ldots, \varphi_{n(x)}(x)\right\}$.

Under these assumptions, we give sufficient conditions for a subspace $H$ of $E$ to be a Korovkin subspace for $T$ and $\tau$. 
THEOREM 3.2. Let $(E, \tau)$ be a regular vector lattice on a locally compact Hausdorff space $X, T \in \mathcal{L}_{\tau}^{+}(E)$ a finitely defined operator of order $n$ with a representation (3.1), and $H$ a subspace of $E$. Assume that:

(i) For every $x \in X$ there exist $h_{1}, \ldots, h_{n(x)} \in H$ such that

$$
\operatorname{det}\left(h_{i}\left(\varphi_{j}(x)\right)\right)_{1 \leq i, j \leq n(x)} \neq 0 .
$$

(ii) For every $x \in X$, every $\varepsilon>0$ and every compact subset $K$ of $X$ satisfying $K \cap\left\{\varphi_{1}(x), \ldots, \varphi_{n}(x)\right\}=\emptyset$, there exists $h \in H$ such that $h \geq 0, h \geq 1$ on $K$ and $h\left(\varphi_{j}(x)\right)<\varepsilon$ for every $j=1, \ldots, n$.

Then $H$ is a Korovkin subspace in $E$ for $T$ and $\tau$.

Proof. By Corollary 2.3, it suffices to show that $\partial_{\tau, H}^{T} X=X$. To this end, choose $x \in X$ and $\mu \in M_{\tau, x}^{T}(H)$; we will prove that $\mu=\sum_{j=1}^{n} \lambda_{j}(x) \varepsilon_{\varphi_{j}(x)}$.

We note first that $\operatorname{supp}(\mu) \subset\left\{\varphi_{1}(x), \ldots, \varphi_{n(x)}(x)\right\}$. Indeed, if $y \in \operatorname{supp}(\mu)$ with $y \notin\left\{\varphi_{1}(x), \ldots, \varphi_{n(x)}(x)\right\}$, then for a given compact neighborhood $K$ of $y$ such that $K \cap\left\{\varphi_{1}(x), \ldots, \varphi_{n(x)}(x)\right\}=\emptyset$, there would exist $f \in C_{\mathrm{c}}(X, \mathbb{R})$, $0 \leq f \leq 1$, such that $\operatorname{supp}(f) \subset K$ and $\int f d \mu>0$. Hence, given $\varepsilon>0$, there would exist $h \in H$ with $h \geq 0, h \geq 1$ on $K$ and $h\left(\varphi_{j}(x)\right)<\varepsilon$ for every $j=1, \ldots, n$. Consequently, $f \leq h$ and

$$
0<\int f d \mu \leq \int h d \mu=T(h)(x)=\sum_{j=1}^{n} \lambda_{j}(x) h\left(\varphi_{j}(x)\right)<\varepsilon \sum_{j=1}^{n} \lambda_{j}(x),
$$

which is impossible, since $\varepsilon>0$ was arbitrarily chosen.

Accordingly, $\operatorname{supp}(\mu) \subset\left\{\varphi_{1}(x), \ldots, \varphi_{n(x)}(x)\right\}$ and thus there exist $\beta_{1}$, $\ldots, \beta_{n(x)} \in \mathbb{R}^{+}$such that $\mu=\sum_{j=1}^{n(x)} \beta_{j} \varepsilon_{\varphi_{j}(x)}$. By assumption, there exist $h_{1}, \ldots, h_{n(x)} \in H$ with $\operatorname{det}\left(h_{i}\left(\varphi_{j}(x)\right)\right)_{1 \leq i, j \leq n(x)} \neq 0$, so that for every $i=$ $1, \ldots, n(x)$ we get

$$
\sum_{j=1}^{n} \lambda_{j}(x) h_{i}\left(\varphi_{j}(x)\right)=T\left(h_{i}\right)(x)=\int h_{i} d \mu=\sum_{j=1}^{n(x)} \beta_{j} h_{i}\left(\varphi_{j}(x)\right) .
$$

Hence, for every $i=1, \ldots, n(x)$,

$$
\sum_{j=1}^{n(x)}\left(\lambda_{j}(x)-\beta_{j}+\sum_{\substack{m_{j} \in\{n(x)+1, \ldots, n\} \\ \varphi_{m_{j}}(x)=\varphi_{j}(x)}} \lambda_{m_{j}}(x)\right) h_{i}\left(\varphi_{j}(x)\right)=0 .
$$

But the system

$$
\sum_{j=1}^{n(x)} h_{i}\left(\varphi_{j}(x)\right) \alpha_{j}=0, \quad i=1, \ldots, n(x),
$$

has $\alpha_{1}=\cdots=\alpha_{n(x)}=0$ as its only solution; consequently, we have $\mu=$ $\sum_{j=1}^{n} \lambda_{j}(x) \varepsilon_{\varphi_{j}(x)}$, and this completes the proof. 
In what follows we provide some methods to construct Korovkin subspaces for finitely defined operators. First, we give some consequences of Theorem 3.2.

COROLlary 3.3. Let $(E, \tau)$ be a regular vector lattice on a locally compact Hausdorff space $X, T \in \mathcal{L}_{\tau}^{+}(E)$ a finitely defined operator of order $n$ with a representation (3.1), and $H$ a subspace of $E$. Assume that

(i) For every $x \in X$ there exist $h_{1}, \ldots, h_{n(x)} \in H$ such that

$$
\operatorname{det}\left(h_{i}\left(\varphi_{j}(x)\right)\right)_{1 \leq i, j \leq n(x)} \neq 0 .
$$

(ii) $^{\prime}$ For every $x \in X$ and every $y \in X \backslash\left\{\varphi_{1}(x), \ldots, \varphi_{n}(x)\right\}$, there exists $h \in H$ such that $h \geq 0, h(y)>0$ and $h\left(\varphi_{i}(x)\right)=0$ for every $i=1, \ldots, n$.

Then $H$ is a Korovkin subspace in $E$ for $T$ and $\tau$.

Proof. We verify assumption (ii) of Theorem 3.2. To this end, fix $x \in X$ and a compact subset $K$ of $X$ satisfying $K \cap\left\{\varphi_{1}(x), \ldots, \varphi_{n}(x)\right\}=\emptyset$; then, for every $y \in K$ there exists $h \in H, h \geq 0$, such that $h\left(\varphi_{i}(x)\right)=0$ for every $i=1, \ldots, n$ and $h(y)>0$. There exists a neighborhood $V_{y}$ of $y$ such that $h(z)>0$ for every $z \in V_{y}$, so that, by the compactness of $K$, there exist $y_{1}, \ldots, y_{m} \in K$ such that $K \subset V_{y_{1}} \cup \cdots \cup V_{y_{m}}$.

Consequently, we can find $m$ positive functions $k_{1}, \ldots, k_{m} \in H$, with $k_{l}\left(\varphi_{i}(x)\right)=0$ for every $i=1, \ldots, n$ and $l=1, \ldots, m$, satisfying the following condition:

for every $y \in K$ there exists $l \in\{1, \ldots, m\}$ such that $k_{l}(y)>0$.

Hence, if we set $k_{0}:=\sum_{l=1}^{m} k_{l}$ and $\alpha:=\min _{y \in K} k_{0}(y)>0$, then the function $h:=(1 / \alpha) k_{0}$ satisfies (ii) of Theorem 3.2 for every $\varepsilon>0$.

If $f_{0} \in E$ and $S$ is a subset of $E$, we set

$$
f_{0} S^{n}:=\left\{f_{0} \cdot g^{n} \mid g \in S\right\} \quad(n \geq 1) .
$$

COROLlary 3.4. Let $(E, \tau)$ be a regular vector lattice on a locally compact Hausdorff space $X$ and $T \in \mathcal{L}_{\tau}^{+}(E)$ a finitely defined operator of order $n$ with a representation (3.1). Moreover, suppose that there exist a strictly positive function $f_{0} \in E$ and a subset $S$ of $E$ such that $f_{0} S \cup f_{0} S^{2} \cup \cdots \cup f_{0} S^{2 n}$ $\subset E$ and, in addition, the following conditions are satisfied:

(i) For every $x \in X$ there exists $h_{0} \in S$ such that $h_{0}\left(\varphi_{i}(x)\right) \neq 0$ for every $i=1, \ldots, n$ and, if $n>1, h_{0}\left(\varphi_{i}(x)\right) \neq h_{0}\left(\varphi_{j}(x)\right)$ whenever $\varphi_{i}(x) \neq \varphi_{j}(x)$.

(ii) For any $x, y \in X$ with $y \notin\left\{\varphi_{1}(x), \ldots, \varphi_{n}(x)\right\}$, there exists $h \in S$ such that $h(y) \neq h\left(\varphi_{i}(x)\right)$ for every $i=1, \ldots, n$.

Then the subspace $H$ generated by $\left\{f_{0}\right\} \cup f_{0} S \cup \cdots \cup f_{0} S^{2 n}$ is a Korovkin subspace in $E$ for $T$ and $\tau$. 
Proof. We show that $H$ satisfies (i) and (ii)' of Corollary 3.3. Fix $x \in X$ and assume that, up to a suitable permutation, $\left\{\varphi_{1}(x), \ldots, \varphi_{n(x)}(x)\right\}$ is the set of all distinct points of $\left\{\varphi_{1}(x), \ldots, \varphi_{n}(x)\right\}$. We shall prove that there exist $h_{1}, \ldots, h_{n(x)} \in H$ such that $\operatorname{det}\left(h_{i}\left(\varphi_{j}(x)\right)\right)_{1 \leq i, j \leq n(x)} \neq 0$.

Indeed, by assumption, there exists $h_{0} \in S$ such that $h_{0}\left(\varphi_{i}(x)\right) \neq 0$ for every $i=1, \ldots, n$ and, if $n>1, h_{0}\left(\varphi_{i}(x)\right) \neq h_{0}\left(\varphi_{j}(x)\right)$ for every $i, j=$ $1, \ldots, n(x), i \neq j$. Consequently, if we set $h_{1}=h_{0}$ and $h_{i}:=f_{0} h_{0}^{i}$ for $1<i \leq n(x)$, we get

$$
\begin{aligned}
\operatorname{det}\left(h_{i}\left(\varphi_{j}(x)\right)\right)_{1 \leq i, j \leq n(x)} & \\
= & f_{0}\left(\varphi_{1}(x)\right) \cdots f_{0}\left(\varphi_{n(x)}(x)\right) h_{0}\left(\varphi_{1}(x)\right) \cdots h_{0}\left(\varphi_{n(x)}(x)\right) \\
& \times \prod_{1 \leq i<j \leq n(x)}\left(h_{0}\left(\varphi_{i}(x)\right)-h_{0}\left(\varphi_{j}(x)\right)\right) \neq 0 .
\end{aligned}
$$

Moreover, for every $y \in X \backslash\left\{\varphi_{1}(x), \ldots, \varphi_{n}(x)\right\}$, there exists $h \in S$ such that $h(y) \neq h\left(\varphi_{i}(x)\right)$ for every $i=1, \ldots, n$. Choose a polynomial $p$ of degree less than or equal to $n$ satisfying $p(h(y))=1$ and $p\left(h\left(\varphi_{i}(x)\right)\right)=0$ for every $i=1, \ldots, n$. Then the function $k:=f_{0} p^{2}(h)$ satisfies (ii)' of Corollary 3.3 for every $\varepsilon>0$, and this completes the proof.

The next result gives sufficient conditions for assumption (i) of Theorem 3.2 (and of Corollary 3.3) to hold.

Lemma 3.5. Let $X$ be a locally compact Hausdorff space, $H$ a subspace of $\mathbb{R}^{X}$, and $\left\{x_{1}, \ldots, x_{m}\right\}$ a set of distinct points of $X, m \geq 2$. If for every $l=1, \ldots, m$ and every $\varepsilon>0$ there exists $k \in H$ satisfying $k \geq 0, k\left(x_{l}\right) \geq 1$ and $k\left(x_{i}\right)<\varepsilon$ for every $i=1, \ldots, m, i \neq l$, then there exist $h_{1}, \ldots, h_{m} \in H$ such that $\operatorname{det}\left(h_{i}\left(x_{j}\right)\right)_{1 \leq i, j \leq m} \neq 0$.

Proof. If $l=1$, then by assumption given $\varepsilon>0$, there exists $h_{1} \in H$ such that $h_{1} \geq 0, h_{1}\left(x_{1}\right) \geq 1$ and $h_{1}\left(x_{i}\right)<\varepsilon$ for every $i=2, \ldots, m$; hence $h_{1}\left(x_{1}\right) \neq 0$.

Let now $1 \leq l<m$ be such that there exist $h_{1}, \ldots, h_{l} \in H$ with $\operatorname{det}\left(h_{i}\left(x_{j}\right)\right)_{1 \leq i, j \leq l} \neq 0$. We proceed to prove that there exists $h_{l+1} \in H$ such that $\operatorname{det}\left(h_{i}\left(x_{j}\right)\right)_{1 \leq i, j \leq l+1} \neq 0$. Otherwise, for every $h \in H$ we would have

$$
\left|\begin{array}{llll}
h_{1}\left(x_{1}\right) & h_{1}\left(x_{2}\right) & \cdots & h_{1}\left(x_{l+1}\right) \\
h_{2}\left(x_{1}\right) & h_{2}\left(x_{2}\right) & \cdots & h_{2}\left(x_{l+1}\right) \\
\vdots & \vdots & \cdots & \vdots \\
h\left(x_{1}\right) & h\left(x_{2}\right) & \cdots & h\left(x_{l+1}\right)
\end{array}\right|=0 .
$$

By expanding this determinant with respect to the last row, there would exist $\alpha_{1}, \ldots, \alpha_{l+1} \in \mathbb{R}$, with $\alpha_{l+1}=\operatorname{det}\left(h_{i}\left(x_{j}\right)\right)_{1 \leq i, j \leq l} \neq 0$, such that $\sum_{i=1}^{l+1} \alpha_{i} h\left(x_{i}\right)=0$ for every $h \in H$. 
Note that $\sum_{i=1}^{n}\left|\alpha_{i}\right|>0$. Indeed, by assumption, given $\varepsilon>0$, there exists $k \in H$ such that $k \geq 0, k\left(x_{i}\right)<\varepsilon$ for every $i=1, \ldots, l$ and $k\left(x_{l+1}\right) \geq 1$. If $\alpha_{1}=\cdots=\alpha_{n}=0$ we would have

$$
\alpha_{l+1} k\left(x_{l+1}\right)=-\sum_{i=1}^{l} \alpha_{i} k\left(x_{i}\right)=0
$$

and thus $k\left(x_{l+1}\right)=0$ contrary to assumption, since $\alpha_{l+1} \neq 0$.

To finish the proof, for every $\varepsilon>0$ and $k \in H$ as above we get

$$
\begin{aligned}
\left|\alpha_{l+1}\right| & \leq\left|\alpha_{l+1}\right| k\left(x_{l+1}\right)=\left|-\sum_{i=1}^{l} \alpha_{i} k\left(x_{i}\right)\right| \\
& \leq \sum_{i=1}^{l}\left|\alpha_{i}\right| k\left(x_{i}\right)<\varepsilon \sum_{i=1}^{l}\left|\alpha_{i}\right| .
\end{aligned}
$$

Since $\varepsilon>0$ was arbitrarily chosen, we conclude that $\alpha_{l+1}=0$, in contradiction with our assumption.

Our statement now follows by induction.

Given $n \in \mathbb{N}$, we denote by $\mathcal{F}_{n}^{\tau}(X)$ the set of all $(\tau, \tau)$-continuous finitely defined operators of order $n$ with a representation (3.1) such that for every $x \in X$ the set $\left\{\varphi_{1}(x), \ldots, \varphi_{n}(x)\right\}$ consists of $n$ distinct points of $X$.

Definition 3.6. Let $(E, \tau)$ be a regular vector lattice on a locally compact Hausdorff space $X$, and $H$ a subspace of $E$. Then $H$ is said to be a Korovkin subspace of order $n$ in $E$ for $\tau$ if $H$ is a Korovkin subspace in $E$ for $\tau$ and for every finitely defined operator $T \in \mathcal{F}_{n}^{\tau}(X)$.

The next results give some methods to construct Korovkin subspaces of order $n$ in $E$.

TheOREM 3.7. Let $X$ be a locally compact Hausdorff space with at least $n+1$ points, $(E, \tau)$ a regular vector lattice on $X$, and $H$ a subspace of $E$. Assume that

(*) For every set of distinct points $x_{1}, \ldots, x_{n} \in X$, every compact subset $K$ of $X$ satisfying $K \cap\left\{x_{1}, \ldots, x_{n}\right\}=\emptyset$ and every $\varepsilon>0$ there exists $h \in H$ such that $h \geq 0, h \geq 1$ on $K$ and $h\left(x_{i}\right)<\varepsilon$ for every $i=$ $1, \ldots, n$.

Then $H$ is a Korovkin subspace of order $n$ in $E$ for $\tau$.

Proof. Fix $T \in \mathcal{F}_{n}^{\tau}(X)$. We verify assumptions (i) and (ii) of Theorem 3.2. Indeed, (ii) obviously follows from $(*)$.

Moreover, for given $l=1, \ldots, n$ and $\varepsilon>0$, fix $\bar{x} \in X \backslash\left\{\varphi_{1}(x), \ldots, \varphi_{n}(x)\right\}$; then, by applying $(*)$ to $K:=\left\{\varphi_{l}(x)\right\}$ and to the subset $\left\{\varphi_{1}(x), \ldots, \varphi_{l-1}(x)\right.$, $\left.\bar{x}, \varphi_{l+1}(x), \ldots, \varphi_{n}(x)\right\}$ of $X$, there exists $k \in H, k \geq 0$, such that $k\left(\varphi_{l}(x)\right)$ 
$\geq 1$ and $k\left(\varphi_{i}(x)\right)<\varepsilon$ for every $i=1, \ldots, n, i \neq l$; by Lemma 3.5, this completes the proof.

COROLlary 3.8. Let $X$ be a locally compact Hausdorff space with at least $n+1$ points, $(E, \tau)$ a regular vector lattice on $X$, and $H$ a subspace of E. Assume that

(**) For every set of distinct points $x_{1}, \ldots, x_{n} \in X$, every $\varepsilon>0$ and every $x \in X \backslash\left\{x_{1}, \ldots, x_{n}\right\}$, there exists $h \in H$ such that $h \geq 0, h(x)>0$ and $h\left(x_{1}\right)=\cdots=h\left(x_{n}\right)=0$.

Then $H$ is a Korovkin subspace of order $n$ in $E$ for $\tau$.

Proof. We verify $(*)$ of Theorem 3.7. To this end, fix distinct $x_{1}, \ldots, x_{n}$ $\in X$ and a compact subset $K$ of $X$ satisfying $K \cap\left\{x_{1}, \ldots, x_{n}\right\}=\emptyset$. Arguing as in the proof of Corollary 3.3, we can find $h_{1}, \ldots, h_{m} \in H, h_{j} \geq 0, h_{j}\left(x_{1}\right)=$ $\cdots=h_{j}\left(x_{n}\right)=0$ for every $j=1, \ldots, m$, such that for every $x \in K$ there exists $j=1, \ldots, m$ with $h_{j}(x)>0$.

Accordingly, if we set $h_{0}:=\sum_{j=1}^{m} h_{j}$ and $\alpha:=\min _{x \in K} h_{0}(x)>0$, then the function $h:=(1 / \alpha) h_{0} \in H$, satisfies $(*)$.

Corollary 3.9. Let $X$ be a locally compact Hausdorff space with at least $n+1$ points, and $(E, \tau)$ a regular vector lattice on $X$. Moreover, suppose that there exist a subset $S$ of $E$ and a strictly positive function $f_{0} \in E$ such that $f_{0} S \cup \cdots \cup f_{0} S^{2 n} \subset E$, where $f_{0} S^{i}$ is defined by (3.2) for every $i=1, \ldots, 2 n$ and, in addition,

(***) For any distinct $x_{1}, \ldots, x_{n} \in X$ and $x \in X \backslash\left\{x_{1}, \ldots, x_{n}\right\}$, there exists $h \in S$ such that $h(x) \neq h\left(x_{i}\right)$ for every $i=1, \ldots, n$.

Then the subspace $H$ generated by $\left\{f_{0}\right\} \cup f_{0} S \cup \cdots \cup f_{0} S^{2 n}$ is a Korovkin subspace of order $n$ in $E$ for $\tau$.

Proof. We verify (**) of Corollary 3.8. Indeed, given distinct $x_{1}, \ldots, x_{n}$ of $X$ and $x \in X \backslash\left\{x_{1}, \ldots, x_{n}\right\}$, by assumption, there exists $h \in S$ such that $h(x) \neq h\left(x_{i}\right)$ for every $i=1, \ldots, n$. Let $P$ be a polynomial of degree less than or equal to $n$ with $P(h(x))=1$ and $P\left(h\left(x_{i}\right)\right)=0$ for every $i=1, \ldots, n$. Then the function $k:=f_{0} P(h)^{2} \in H$ satisfies $(* *)$ for every $\varepsilon>0$.

ExAMPLES 3.10. 1. Let $X$ be a locally compact Hausdorff space, $(E, \tau)$ a regular vector lattice, and assume that there exists a strictly positive one-to-one function $f_{0} \in E$ such that $f_{0}^{2}, \ldots, f_{0}^{2 n+1} \in E$. Then the subspace generated by $\left\{f_{0}, f_{0}^{2}, \ldots, f_{0}^{2 n+1}\right\}$ is a Korovkin subspace of order $n$ in $E$ for $\tau$.

For example, let $X$ be an interval of $\mathbb{R}$. Then, for every $\alpha \neq 0$, the subspace generated by $\left\{e^{\alpha x}, \ldots, e^{(2 n+1) \alpha x}\right\}$ is a Korovkin subspace of order $n$ in every regular vector sublattice of $C(X, \mathbb{R})$ containing these functions. 
Let $X$ be a subinterval of $] 0,+\infty[$. Then the subspace generated by $\left\{x, x^{2}, \ldots, x^{2 n+1}\right\}$ is a Korovkin subspace of order $n$ in every regular vector sublattice of $C(X, \mathbb{R})$ containing these functions.

Let $X$ be a subinterval of $[1,+\infty[$. Then the subspace generated by $\left\{x^{-1}, \ldots, x^{-2 n-1}\right\}$ is a Korovkin subspace of order $n$ in every regular vector sublattice of $C(X, \mathbb{R})$ containing these functions.

2. Set $f_{0}:=\mathbf{1}$ and $f_{1}(x)=x$ for every $x \in \mathbb{R}$; Corollary 3.9 applied to $f_{0}$ and $S:=\left\{f_{1}\right\}$ shows that the subspace generated by $\left\{\mathbf{1}, x, x^{2}, \ldots, x^{2 n}\right\}$ is a Korovkin subspace of order $n$ in every regular vector sublattice of $C(X, \mathbb{R})$ containing these functions.

The previous examples show that finitely defined operators, under suitable assumptions, admit finite-dimensional Korovkin subspaces. We conclude this section with a result which ensures that they are the only positive linear operators with this property.

Proposition 3.11. Let $(E, \tau)$ be a regular vector lattice on a locally compact Hausdorff space $X, H$ an $n$-dimensional subspace of $E, n \geq 1$ and $T \in \mathcal{L}_{\tau, \tau_{\mathrm{s}}}^{+}(E)($ see $[3,(3.8)])$. Then there exist $\lambda_{1}, \ldots, \lambda_{n+2} \in \mathbb{R}^{X}$ with $\lambda_{i} \geq 0$ for every $i=1, \ldots, n+2$, and $\varphi_{1}, \ldots, \varphi_{n+2}: X \rightarrow X$ such that

$$
T(h)=\sum_{i=1}^{n+2} \lambda_{i}\left(h \circ \varphi_{i}\right) \quad \text { for every } h \in H .
$$

Moreover, if $T \in \mathcal{L}_{\tau}^{+}(E)$ and $H$ is a Korovkin subspace in $E$ for $T$ and $\tau$, then

$$
T(f)=\sum_{i=1}^{n+2} \lambda_{i}\left(f \circ \varphi_{i}\right) \quad \text { for every } f \in E .
$$

Proof. The first part follows by applying [11, Proposition 2.1] and [3, Lemma 2.4] to each continuous positive linear functional $\mu_{x}$ on $E(x \in X)$ defined by $\mu_{x}(f):=T(f)(x)(f \in E)$. The second part is a consequence of Corollary 2.3 .

4. Korovkin closure for the identity operator. In this section, applying the results of Section 2, we characterize the Korovkin closure of a subspace of a regular vector lattice for the identity operator $I$ on $E$. We also describe several methods to construct Korovkin subspaces for the identity operator and hence for every continuous lattice homomorphism $T \in$ $\mathcal{L}_{\tau}^{+}(E)$. These methods will be used to exhibit examples of finite-dimensional Korovkin subspaces for the identity operator.

To this end, we recall the following specialization of Definition 2.1.

Definition 4.1. Let $(E, \tau)$ be a regular vector lattice on a locally compact Hausdorff space $X$, and $H$ a subspace of $E$. The subspace of $E$ defined 
by

$\operatorname{Kor}(H)_{\tau}:=\left\{f \in E \mid\right.$ if $\left(L_{i}\right)_{i \in I}^{\leq}$is a $\tau$-equicontinuous net of positive linear operators from $E$ into $E$ satisfying

$$
\begin{aligned}
& \lim _{i \in I} \leq L_{i}(h)=h \text { in }(E, \tau) \text { for every } h \in H, \text { then } \\
& \left.\lim _{i \in I} \leq L_{i}(f)=f \text { in }(E, \tau)\right\}
\end{aligned}
$$

is said to be the Korovkin closure of $H$ in $E$ for $\tau$ (and for the operator $I)$.

Moreover, $H$ is said to be a Korovkin subspace in $E$ for $\tau$ (and for the operator $I$ ) if $\operatorname{Kor}(H)_{\tau}=E$.

The following consequence of Theorem 2.2 gives a characterization of the Korovkin closure of $H$ in $E$ for $\tau$.

THEOREM 4.2. Let $(E, \tau)$ be a regular vector lattice on a locally compact Hausdorff space $X$, and $H$ a subspace of $E$. Then

$$
\begin{aligned}
\operatorname{Kor}(H)_{\tau} & =\widehat{H}_{\tau} \\
& =\left\{f \in E \mid \int f d \mu=f(x) \text { for every } x \in X \text { and } \mu \in M_{\tau, x}(H)\right\},
\end{aligned}
$$

where $\widehat{H}_{\tau}$ and $M_{\tau, x}(H)$ are defined by (4.1) and (4.2) of [3], respectively.

Korovkin subspaces for $\tau$ are characterized as follows.

Corollary 4.3. Under the assumptions of Theorem 4.2, $\mathrm{H}$ is a Korovkin subspace in $E$ for $\tau$ if and only if $\partial_{\tau, H} X=X$, where $\partial_{\tau, H} X$ is defined by $[3,(4.3)]$. In particular, if $\partial_{\tau, H} X=X$, then $H$ is also a Korovkin subspace in $E$ for $\tau$ and for every lattice homomorphism $T \in \mathcal{L}_{\tau}^{+}(E)$.

Proof. This is a consequence of Corollary 2.3 and Corollaries 4.2 and 4.6 of $[3]$.

In what follows, we find simpler criteria to establish whether a subspace of $E$ is a Korovkin subspace in $E$ and hence for every lattice homomorphism $T \in \mathcal{L}_{\tau}^{+}(E)$. To this end, we introduce the following definition (see $[1$, Section $2.6])$.

Definition 4.4. Let $(E, \tau)$ be a regular vector lattice on a locally compact Hausdorff space $X$ and $H$ a subspace of $E$.

A point $x_{0} \in X$ is said to be an $H$-peak-point if:

(i) There exists $h \in H$ such that $h\left(x_{0}\right) \neq 0$.

(ii) For every $y \in X, y \neq x_{0}$, there exists $k \in H$ such that $k \geq 0$, $k\left(x_{0}\right)=0$ and $k(y)>0$.

We denote by $\Gamma_{H} X$ the set of all $H$-peak-points of $X$. 
As a matter of fact, we shall prove that $\Gamma_{H} X$ is a subset of $\partial_{\tau, H} X$; consequently, by Corollary 4.3, to show that $H$ is a Korovkin subspace for $\tau$, it is enough to prove that $\Gamma_{H} X=X$. First, we state a preliminary lemma (see [5, Section 2]).

Lemma 4.5. Let $(E, \tau)$ be a regular vector lattice on a locally compact Hausdorff space $X, x \in X$, and $H$ a subspace of $E$. Suppose that:

(i) For every $\varepsilon>0$ and every compact subset $K$ of $X$ with $K \cap\{x\}=\emptyset$ there exists $u \in H$ such that $u \geq 0, u \geq 1$ on $K$ and $u(x)<\varepsilon$;

(ii) There exists $h \in H$ such that $h(x) \neq 0$.

Then $x \in \partial_{\tau, H} X$.

Proof. Fix a measure $\mu \in M_{\tau, x}(H)$; we will prove that $\mu=\varepsilon_{x}$.

Arguing as in the proof of Theorem 3.2, one can show that $\operatorname{supp}(\mu) \subset\{x\}$ and hence $\mu=\lambda \varepsilon_{x}$ with $\lambda \geq 0$. Since there exists $h \in H$ such that $h(x) \neq 0$, we get

$$
h(x)=\int h d \mu=\lambda h(x),
$$

and this completes the proof.

We are now in a position to prove the following result (see $[1$, Theorem $2.2 \cdot 6])$.

THEOREM 4.6. Let $(E, \tau)$ be a regular vector lattice on a locally compact Hausdorff space $X$, and $H$ a subspace of $E$. Then $\Gamma_{H} X \subset \partial_{\tau, H} X$. Consequently, if $\Gamma_{H} X=X$, then $H$ is a Korovkin subspace in $E$ for $\tau$ and for both the identity operator on $E$ and every lattice homomorphism $T \in \mathcal{L}_{\tau}^{+}(E)$.

Proof. Fix $x \in \Gamma_{H} X$. In order to show that $x \in \partial_{\tau, H} X$, we prove that $x$ satisfies conditions (i) and (ii) of Lemma 4.5.

Indeed, for a given compact subset $K$ of $X$ such that $K \cap\{x\}=\emptyset$, by applying the same reasoning of the proof of Corollary 3.3, one can show that there exist $h_{1}, \ldots, h_{n} \in H$ with $h_{i} \geq 0$ and $h_{i}(x)=0$ for every $i=1, \ldots, n$, and with the following property:

for every $y \in K$ there exists $i \in\{1, \ldots, n\}$ such that $h_{i}(y)>0$.

If we set $h_{0}:=\sum_{i=1}^{n} h_{i}$ and $\alpha:=\min _{y \in K} h_{0}(y)>0$, the function $u:=$ $(1 / \alpha) h_{0}$ clearly satisfies (i) of Lemma 4.5 for every $\varepsilon>0$. Moreover, since $x \in \Gamma_{H} X$, (ii) also holds, so that $x \in \partial_{\tau, H} X$.

The second part is a consequence of Corollary 4.3 and of [3, Corollary 4.6].

From Theorem 4.6 or from Corollary 3.9 for $n=1$, one can deduce the following results. 
Proposition 4.7. Let $(E, \tau)$ be a regular vector lattice on a locally compact Hausdorff space $X$. Assume that there exist a strictly positive function $f_{0} \in E$ and a subset $S$ of $E$ separating the points of $X$ such that $f_{0} S, f_{0} S^{2} \subset E$, where $f_{0} S^{i}$ is defined by (3.2) for $i=1,2$. Then the subspace generated by $\left\{f_{0}\right\} \cup f_{0} S \cup f_{0} S^{2}$ is a Korovkin subspace in $E$ for $\tau$.

Proposition 4.8. Let $X$ be a locally compact Hausdorff space and $(E, \tau)$ a regular vector lattice on $X$. Assume that there exist a strictly positive function $f_{0} \in E$ and a finite subset $S:=\left\{h_{1}, \ldots, h_{n}\right\}$ of $E$ separating the points of $X$ such that $f_{0} S, f_{0} S^{2} \subset E$. Then the subspace $H$ generated by $\left\{f_{0}, f_{0} h_{1}, \ldots, f_{0} h_{n}, f_{0} \sum_{i=1}^{n} h_{i}^{2}\right\}$ is a Korovkin subspace in $E$ for $\tau$.

Proof. Fix distinct $x, y \in X$; by assumption, there exists $i \in\{1, \ldots, n\}$ such that $h_{i}(x) \neq h_{i}(y)$. Since the function

$$
k:=f_{0} \sum_{i=1}^{n}\left(h_{i}-h_{i}(x)\right)^{2} \in H
$$

satisfies condition (ii) of Definition 4.4, we have $x \in \Gamma_{H} X$; hence, our statement follows from Theorem 4.6.

In addition to Examples 3.10, with $n=1$, we now list some other examples of Korovkin subspaces for $\tau$ and either for $I$ or for every lattice homomorphism $T \in \mathcal{L}_{\tau}^{+}(E)$. Other examples in the setting of $C_{0}(X, \mathbb{R})$ or $C(X, \mathbb{R})$, with $X$ compact, can be found in [1, Ch. 4 and Appendix C].

ExAmples 4.9. 1. Let $X$ be an interval of $\mathbb{R}$. Then the subspace generated by $\left\{\mathbf{1}, x, x^{2}\right\}$ is a Korovkin subspace in every regular vector sublattice of $C(X, \mathbb{R})$ containing these functions.

2 . Let $X$ be a locally compact subset of $\mathbb{R}^{p}, p \geq 1$, and for every $i=$ $1, \ldots, p$, consider the mapping $\operatorname{pr}_{i}: X \rightarrow \mathbb{R}$ defined by $\operatorname{pr}_{i}(x):=x_{i}$ for $x=\left(x_{1}, \ldots, x_{p}\right) \in X$. Then the subspace generated by

$$
\left\{\mathbf{1}, \mathrm{pr}_{1}, \ldots, \mathrm{pr}_{p}, \sum_{i=1}^{p} \operatorname{pr}_{i}^{2}\right\}
$$

is a Korovkin subspace in every regular vector sublattice of $C(X, \mathbb{R})$ containing these functions.

3. Proposition 4.8 applied to the mappings $f_{0}(x):=\exp \left(-\|x\|^{2}\right)$ and $h_{1}(x):=x_{1} \exp \left(-\|x\|^{2}\right), \ldots, h_{n}(x):=x_{n} \exp \left(-\|x\|^{2}\right), h_{n+1}(x)=f_{0}(x), x=$ $\left(x_{1}, \ldots, x_{n}\right) \in \mathbb{R}^{n}$ shows that the subspace generated by

$$
\begin{aligned}
\left\{\exp \left(-\|x\|^{2}\right), \exp \left(-2\|x\|^{2}\right), x_{1} \exp \left(-2\|x\|^{2}\right), \ldots\right. \\
\left.\ldots, x_{n} \exp \left(-2\|x\|^{2}\right),\left(1+\|x\|^{2}\right) \exp \left(-3\|x\|^{2}\right)\right\}
\end{aligned}
$$

is a Korovkin subspace in every regular vector sublattice of $C(X, \mathbb{R})$ containing these functions. 
We now give an application of these results. For every $\alpha>0$ denote by $E_{\alpha}$ the subspace of all $f \in C([0,+\infty[, \mathbb{R})$ such that

$$
\|f\|_{\alpha}:=\sup _{x \geq 0} e^{-\alpha x}|f(x)|<+\infty .
$$

Moreover, set

$$
E_{\alpha}^{0}:=\left\{f \in C \left(\left[0,+\infty[, \mathbb{R})\left|\lim _{x \rightarrow+\infty} e^{-\alpha x}\right| f(x) \mid=0\right\} \subset E_{\alpha} .\right.\right.
$$

Clearly, for every $0<\alpha<\beta$ we have

$$
E_{\alpha} \subset E_{\beta}^{0} \text { and }\|\cdot\|_{\beta} \leq\|\cdot\|_{\alpha} \text { on } E_{\alpha} .
$$

Set

$$
E_{\infty}:=\bigcup_{\alpha>0} E_{\alpha}, \quad E:=\bigcap_{\alpha>0} E_{\alpha}^{0} .
$$

Then $E$, endowed with the locally convex topology $\tau$ generated by the family $\left(\|\cdot\|_{\alpha}\right)_{\alpha>0}$, is a weighted function space $([3$, Ex. $2.3,3])$ and hence a regular vector lattice.

Fix $\lambda \in C\left(\left[0,+\infty[, \mathbb{R})\right.\right.$ with $0 \leq \lambda \leq \mathbf{1}$, and for every $n \geq 1, f \in E_{\infty}$ and $x \geq 0$ set

$$
\begin{aligned}
& M_{n, \lambda}(f)(x) \\
& \quad:=\sum_{p=0}^{n} \sum_{h=0}^{\infty}\left(\begin{array}{l}
n \\
p
\end{array}\right) \lambda(x)^{p}(1-\lambda(x))^{n-p} e^{-p x} \frac{(p x)^{h}}{h !} f\left(\frac{h+(n-p) x}{n}\right) .
\end{aligned}
$$

These positive operators have been introduced in [4]. It is known that they are well defined on $E_{\infty}$ and, moreover, that $M_{n, \lambda}\left(E_{\infty}\right) \subset E_{\infty}$.

Note also that, for $\lambda=\mathbf{1}$, the operators $M_{n, \mathbf{1}}$ become the classical SzászMirakjan operators defined by

$$
M_{n}(f)(x)=\sum_{h=0}^{\infty} e^{-n x} \frac{(n x)^{h}}{h !} f\left(\frac{h}{n}\right) \quad\left(f \in E_{\infty}, x \geq 0\right) .
$$

In [4, Prop. 1.1,1] it was shown that, if $\alpha>0$ and $f \in E_{\alpha}$ then for every $n \geq 1$,

$$
M_{n, \lambda}(f) \in E_{\gamma} \quad \text { and } \quad\left\|M_{n, \lambda}(f)\right\|_{\gamma} \leq\|f\|_{\alpha},
$$

where $\gamma:=n(\exp ((\alpha / n))-1)>\alpha$.

Proposition 4.10. Let $(E, \tau)$ be the weighted function space (4.2) and $\left(M_{n, \lambda}\right)_{n \geq 1}$ the sequence of positive linear operators (4.3). Then

(i) $M_{n, \lambda}(E) \subset E$ for every $n \geq 1$ and the sequence $\left(M_{n, \lambda}\right)_{n \geq 1}$ is equicontinuous from $(E, \tau)$ into $(E, \tau)$.

(ii) $\lim _{n \rightarrow \infty} M_{n, \lambda}(f)=f$ in $(E, \tau)$ for every $f \in E$. 
Proof. (i) Fix $f \in E$ and $n \geq 1$. Given $\beta>0$, choose $0<\gamma<\beta$; as $f \in E_{\alpha}$ for $\alpha:=n \log (\gamma / n+1)$, from (4.1) and (4.4) it follows that $M_{n, \lambda}(f) \in E_{\gamma} \subset E_{\beta}^{0}$. Accordingly, $M_{n, \lambda}(f) \in E$.

To show that the sequence $\left(M_{n, \lambda}\right)_{n \geq 1}$ is equicontinuous, fix $\beta>0$ and choose again $\gamma>0$ such that $\gamma<\beta$.

Note that the function $\varphi(x):=x \log (\gamma / x+1)(x \geq 1)$ is continuous, strictly positive and has a finite limit as $x \rightarrow+\infty$. Therefore $\varphi$ has a minimum value on $[1,+\infty[$ which is strictly positive. Thus we can consider $\alpha_{0}:=\inf _{n \geq 1} n \log (\gamma / n+1)>0$.

Now for every $f \in E$ and $n \geq 1$, setting $\alpha:=n \log (\gamma / n+1) \geq \alpha_{0}$ and combining (4.1) and (4.4), we obtain

$$
\left\|M_{n, \lambda}(f)\right\|_{\beta} \leq\left\|M_{n, \lambda}(f)\right\|_{\gamma} \leq\|f\|_{\alpha} \leq\|f\|_{\alpha_{0}}
$$

which proves (i).

(ii) We shall use Example 4.9(1): the subspace generated by $\left\{e_{0}, e_{1}, e_{2}\right\}$ is a Korovkin subspace in $E$, where $e_{i}(x):=x^{i}$ for $i=0,1,2$ and $x \geq 0$.

Indeed, we have $M_{n, \lambda}\left(e_{0}\right)=e_{0}, M_{n, \lambda}\left(e_{1}\right)=e_{1}$ and $M_{n, \lambda}\left(e_{2}\right)=e_{2}+$ $(\lambda / n) e_{1}$ for every $n \geq 1$; hence $M_{n, \lambda}\left(e_{k}\right) \rightarrow e_{k}$ in $(E, \tau)$ for $k=0,1,2$ and so the result follows.

5. Korovkin subspaces for positive continuous projections. In a previous paper (see [2]), in the framework of adapted spaces of continuous functions, we have studied a particular class of positive projections, which we have called affine projections; in particular, in that paper we have presented some characterizations of the Choquet boundary of the ranges of these projections and we have determined suitable Korovkin subsets for them.

In what follows, we generalize the results of [2] to the setting of regular vector lattices of continuous functions.

From now on, we fix a locally compact Hausdorff space $X$, a regular vector lattice $(E, \tau)$ on $X$ and a projection $T \in \mathcal{L}_{\tau, \tau_{\mathrm{s}}}^{+}(E)$, i.e. a positive linear $\left(\tau, \tau_{\mathrm{s}}\right)$-continuous operator such that

$$
T(T(f))=T(f) \quad \text { for every } f \in E .
$$

Moreover, we set

$$
\begin{aligned}
& H_{T}:=T(E)=\{f \in E \mid T(f)=f\}, \\
& H_{T}^{2}:=\left\{h^{2} \mid h \in H_{T}\right\} .
\end{aligned}
$$

Below we list some examples of such projections; for further examples, see [2, Examples 2.2].

Examples 5.1. 1. If $X:=] 0,1]$ and $(E, \tau)$, where $\tau \in\left\{\tau_{\mathrm{s}}, \tau_{\mathrm{c}}\right\}$, is a regular vector lattice on $X$ containing the function $e_{1}(x)=x(0<x \leq 1)$, 
then the operator $T: E \rightarrow E$ defined by

$$
T(f)(x):=x f(1) \quad(f \in E, 0<x \leq 1)
$$

is a positive linear $(\tau, \tau)$-continuous projection.

2. If $X:=\left[0,+\infty\left[\right.\right.$ and $(E, \tau)$, where $\tau \in\left\{\tau_{\mathrm{s}}, \tau_{\mathrm{c}}\right\}$, is a regular vector lattice containing the function $e_{2}(x)=\exp (-x)(x \geq 0)$, then the operator $T: E \rightarrow E$ defined by

$$
T(f)(x):=\exp (-x) f(0) \quad(f \in E, x \geq 0)
$$

is a positive linear $(\tau, \tau)$-continuous projection.

From (5.1) it follows that, for a given projection $T \in \mathcal{L}_{\tau, \tau_{\mathrm{s}}}^{+}(E)$, for every $x \in X$ the Borel regular measure $\mu_{x}$ on $X$ (whose existence is guaranteed by $\left[3\right.$, Theorem 2.6]), such that $E \subset \mathcal{L}^{1}\left(E, \mu_{x}\right)$ and

$$
T(f)(x)=\int f d \mu_{x} \quad(f \in E),
$$

is an $H_{T}$-representing measure for $x$ with respect to $\tau$.

$T$ is said to be a sub-markovian operator if $\mu_{x}(X) \leq 1$ for every $x \in X$. $T$ is said to be a markovian operator if $\mu_{x}(X)=1$ for every $x \in X$.

Clearly, if $\mathbf{1} \in E$ and $T(\mathbf{1})=\mathbf{1}$, then $T$ is a markovian operator. The projections in Examples 5.1 are sub-markovian.

In order to characterize the Choquet boundary of $H_{T}$ in terms of the projection $T$, we give a preliminary lemma.

Lemma 5.2. Let $(E, \tau)$ be a regular vector lattice on a locally compact Hausdorff space $X$, and $T \in \mathcal{L}_{\tau, \tau_{\mathrm{s}}}^{+}(E)$ a sub-markovian projection such that $H_{T}^{2} \subset E$, where $H_{T}^{2}$ is defined by (5.2). Then, for every $f \in H_{T}$,

$$
f^{2}(x) \leq T\left(f^{2}\right)(x) \quad(x \in X) .
$$

Proof. Indeed, for every $x \in X$ and $f \in H_{T}$, from the Hölder inequality and (5.3) it follows that

$$
\begin{aligned}
|f(x)| & =|T(f)(x)|=\left|\int f d \mu_{x}\right| \leq \int|f| d \mu_{x} \\
& \leq\left(\int f^{2} d \mu_{x}\right)^{1 / 2}\left(\int \mathbf{1} d \mu_{x}\right)^{1 / 2} \leq\left(T\left(f^{2}\right)(x)\right)^{1 / 2} \quad\left(f \in H_{T}\right) .
\end{aligned}
$$

From now on, we assume that $T \in \mathcal{L}_{\tau, \tau_{\mathrm{s}}}^{+}(E)$ is a positive projection whose range $H_{T}$ separates linearly the points of $X$, i.e. for any distinct $x, y \in X$ there exist $h, k \in H_{T}$ such that $h(x) k(y) \neq h(y) k(x)$.

In this case, for every $x \in X$ there exists $h \in H_{T}$ such that $h(x) \neq 0$.

THEOREM 5.3. Let $(E, \tau)$ be a regular vector lattice on a locally compact Hausdorff space $X$, and $T \in \mathcal{L}_{\tau, \tau_{\mathrm{s}}}^{+}(E)$ a sub-markovian projection whose range $H_{T}$ separates linearly the points of $X$ and satisfies $H_{T}^{2} \subset E$, where 
$H_{T}^{2}$ is defined by (5.2). Then

$$
\partial_{\tau, H_{T}} X=\{x \in X \mid T(f)(x)=f(x) \text { for every } f \in E\} .
$$

If, in addition, $S$ is a subset of $H_{T}$ separating the points of $X$, then

$$
\partial_{\tau, H_{T}} X=\left\{x \in X \mid T\left(f^{2}\right)(x)=f^{2}(x) \text { for every } f \in S\right\} .
$$

Finally, if $\left(u_{n}\right)_{n \geq 1}$ is a sequence in $H_{T}$ separating the points of $X$ such that the series $u:=\sum_{n=1}^{\infty} u_{n}^{2}$ is pointwise convergent on $X$ and $u \in E$, then

$$
\partial_{\tau, H_{T}} X=\{x \in X \mid T(u)(x)=u(x)\} .
$$

Proof. Let $x \in \partial_{\tau, H_{T}} X$; then, clearly, from (5.3) it follows that for every $f \in E$,

$$
T(f)(x)=\int f d \mu_{x}=\int f d \varepsilon_{x}=f(x) .
$$

Conversely, consider $x \in X$ such that $T(f)(x)=f(x)$ for every $f \in E$, i.e. $\mu_{x}=\varepsilon_{x}$. We shall prove that $x \in \partial_{\tau, H_{T}} X$; for this purpose, by Theorem 4.6, we shall show that $x \in \Gamma_{H_{T}} X$.

Indeed, fix $y \in X, y \neq x$. Since $H_{T}$ separates linearly the points of $X$, there exist $h, k \in H_{T}$ such that $h(x) k(y) \neq h(y) k(x)$. Set $p:=T((h(x) k-$ $\left.k(x) h)^{2}\right) \in H_{T}$; then $p \geq 0$,

$$
p(x)=\int(h(x) k-k(x) h)^{2} d \mu_{x}=\int(h(x) k-k(x) h)^{2} d \varepsilon_{x}=0
$$

and, finally, according to Lemma 5.2,

$$
0<(h(x) k(y)-k(x) h(y))^{2} \leq p(y) .
$$

Hence, $x \in \Gamma_{H_{T}} X$, and this completes the proof of (5.5).

Consider now a subset $S$ of $H_{T}$ separating the points of $X$. Since (5.5) holds, in (5.6) we only have to show that if $x \in X$ and $T\left(f^{2}\right)(x)=f^{2}(x)$ for every $f \in S$, then $\mu_{x}=\varepsilon_{x}$.

In fact, for $f \in S$, we get

$$
\begin{aligned}
0 & \leq \int(f-f(x))^{2} d \mu_{x}=\int f^{2} d \mu_{x}-2 f(x) \int f d \mu_{x}+f^{2}(x) \mu_{x}(X) \\
& \leq f^{2}(x)-2 f^{2}(x)+f^{2}(x)=0,
\end{aligned}
$$

so that

$$
\int(f-f(x))^{2} d \mu_{x}=0
$$

hence,

$$
\operatorname{supp}\left(\mu_{x}\right) \subset \bigcap_{f \in S}\{y \in X \mid f(y)=f(x)\}=\{x\} .
$$

Accordingly, there exists $\lambda \geq 0$ such that $\mu_{x}=\lambda \varepsilon_{x}$. Since there exists $h \in H_{T}$ such that $h(x) \neq 0$, we get

$$
h(x)=\int h d \mu_{x}=\lambda h(x)
$$

and consequently $\mu_{x}=\varepsilon_{x}$. 
Finally, in order to prove (5.7), assume that $T(u)(x)=u(x)$ for some $x \in X$. According to the Beppo Levi theorem,

$$
\sum_{n=1}^{\infty} u_{n}^{2}(x)=u(x)=\int u d \mu_{x}=\sum_{n=1}^{\infty} \int u_{n}^{2} d \mu_{x}=\sum_{n=1}^{\infty} T\left(u_{n}^{2}\right)(x) .
$$

Lemma 5.2 shows that $u_{n}^{2} \leq T\left(u_{n}^{2}\right)$ for every $n \geq 1$ and hence

$$
\int u_{n}^{2} d \mu_{x}=u_{n}^{2}(x) \quad \text { for every } n \geq 1 .
$$

From (5.6) applied to $S:=\left\{u_{n} \mid n \geq 1\right\}$, it follows that $x \in \partial_{\tau, H_{T}} X$.

By means of Theorem 5.3 and Corollary 2.3, we can determine some Korovkin subspaces for $(\tau, \tau)$-continuous positive projections on $E$ :

THEOREM 5.4. Let $(E, \tau)$ be a regular vector lattice on a locally compact Hausdorff space $X$, and $T \in \mathcal{L}_{\tau}^{+}(E)$ a sub-markovian projection whose range $H_{T}$ separates linearly the points of $X$ and satisfies $H_{T}^{2} \subset E$, where $H_{T}^{2}$ is defined by (5.2). Assume that there exists a subset $\mathcal{U}_{T}$ of $E$ such that

(i) $u \leq T(u)$ for every $u \in \mathcal{U}_{T}$.

(ii) $\partial_{\tau, H_{T}} X=\left\{x \in X \mid T(u)(x)=u(x)\right.$ for every $\left.u \in \mathcal{U}_{T}\right\}$.

Then the subspace $\widetilde{H}_{T}$ generated by $H_{T} \cup \mathcal{U}_{T}$ is a Korovkin subspace in $E$ for $T$ and $\tau$.

Proof. Applying Corollary 2.3, we shall prove that $\partial_{\tau, \widetilde{H}_{T}}^{T} X=X$. Indeed, for $x \in X$ and $\mu \in M_{\tau, x}^{T}\left(\widetilde{H}_{T}\right)$, we have

$$
\int k d \mu=T(k)(x)=k(x) \quad \text { for every } k \in H_{T}
$$

and

$$
\int u d \mu=T(u)(x) \quad \text { for every } u \in \mathcal{U}_{T},
$$

so that, clearly, $\mu \in M_{\tau, x}\left(H_{T}\right)$.

Furthermore, for $u \in \mathcal{U}_{T}$ we get

$$
\int T(u) d \mu=T(u)(x)=\int u d \mu
$$

and thus $\int(T(u)-u) d \mu=0$. Accordingly,

$$
\operatorname{supp}(\mu) \subset\left\{x \in X \mid T(u)(x)=u(x) \text { for every } u \in \mathcal{U}_{T}\right\}=\partial_{\tau, H_{T}} X .
$$

Hence, taking Theorem 5.3 into account, we get

$$
\int f d \mu=\int T(f) d \mu=T(f)(x)
$$

for every $f \in E$, which completes the proof.

Remarks 5.5. 1. Note that under the assumptions of Theorem 5.4, if $\left(L_{i}\right)_{i \in I}^{\leq}$is a $\tau$-equicontinuous net of positive linear operators from $E$ into $E$ 
such that

$$
\lim _{i \in I} \leq L_{i}(h)=h \quad \text { for every } h \in H \text { in }(E, \tau)
$$

and

$$
\lim _{i \in I} L_{i}(u)=T(u) \quad \text { for every } u \in \mathcal{U}_{T} \text { in }(E, \tau)
$$

one gets

$$
\lim _{i \in I} \leq L_{i}(f)=T(f) \quad \text { for every } f \in E \text { in }(E, \tau) .
$$

2. According to Theorems 5.3 and 5.4, if $S$ is a subset of $H_{T}$ separating the points of $X$, then the subspace generated by $H_{T} \cup S^{2}$ is a Korovkin subspace in $E$ for $T$ and $\tau$.

Moreover, if $\left(u_{n}\right)_{n \geq 1}$ is a sequence in $H_{T}$ separating the points of $X$, such that the series $u:=\sum_{n=1}^{\infty} u_{n}^{2}$ is pointwise convergent in $X$ and $u \in E$, then the subspace generated by $H_{T} \cup\{u\}$ is Korovkin subspace in $E$ for $T$ and $\tau$.

\section{References}

[1] F. Altomare and M. Campiti, Korovkin-Type Approximation Theory and its Applications, de Gruyter Stud. Math. 17, de Gruyter, Berlin, 1994.

[2] F. Altomare and M. Cappelletti Montano, Affine projections on adapted subalgebras of continuous functions, Positivity, to appear.

[3] - - - Korovkin-type theorems in regular locally convex vector lattices of continuous functions-Part I, Studia Math. 171 (2005), 239-260.

[4] F. Altomare and I. Carbone, On a new sequence of positive linear operators on unbounded intervals, Rend. Circ. Mat. Palermo (2) Suppl. 40 (1996), 23-36.

[5] A. Attalienti, Uniqueness subspaces and Stone-Weierstrass theorems for adapted spaces, Math. Japon. 39 (1994), 291-304.

[6] H. Bauer, Theorems of Korovkin type for adapted spaces, Ann. Inst. Fourier (Grenoble) 23 (1973), no. 4, 245-260.

[7] —, Convergence of monotone operators, Math. Z. 136 (1974), 315-330.

[8] H. Bauer and K. Donner, Korovkin approximation in $C_{0}(X)$, Math. Ann. 236 (1978), 225-237.

[9] G. Choquet, Lectures on Analysis, Vols. I-III, Benjamin, New York, 1969.

[10] H. O. Flösser, A Korovkin-type theorem in locally convex M-spaces, Proc. Amer. Math. Soc. 72 (1978), 456-460.

[11] —, Korovkin-closures of finite sets, Arch. Math. (Basel) 32 (1979), 600-608.

[12] H. O. Flösser, R. Irmisch and W. Roth, Infimum-stable convex cones and approximation, Proc. London Math. Soc. 42 (1981), 104-120.

[13] M. W. Grossman, Korovkin theorems for adapted spaces with respect to a positive operator, Math. Ann. 220 (1976), 253-262.

[14] G. Mokobodzki and D. Sibony, Cônes adaptés de fonctions continues et théorie du potentiel, in: Séminaire Choquet 1966/67, Initiation à l'Analyse, Fasc. 1, Exp. 5, Secrétariat Mathématique, Paris, 1968, 35 pp.

[15] L. Nachbin, Elements of Approximation Theory, Van Nostrand, Princeton, NJ, 1967. 
[16] J. B. Prolla, Approximation of Vector-Valued Functions, Math. Stud. 25, NorthHolland, Amsterdam, 1977.

[17] W. Roth, A Korovkin type theorem for weighted spaces of continuous functions, Bull. Austral. Math. Soc. 55 (1997), 239-248.

[18] D. Sibony, Cônes de fonctions et potentiels, lecture notes, McGill Univ., Montreal, 1968.

Department of Mathematics

University of Bari

Campus Universitario

Via E. Orabona, 4

70125 Bari, Italy

E-mail: altomare@dm.uniba.it montano@dm.uniba.it

Received September 22, 2004

Revised version July 21, 2005 